\title{
A successful pregnancy and live birth after defragmented low quality blastocysts transfer (review of 20 clinical cases)
}

\author{
Konstantin V Kirienko ${ }^{1 *}$, Valentina P Apryshko', Vladimir V Voloshanenko ${ }^{1}$, Anna G Mironova ${ }^{1,2}$ and Sergey A Yakovenko ${ }^{1,3}$ \\ ${ }^{1}$ Altravita IVF Clinic, 4A Nagornaya, 117186, Moscow, Russia \\ ${ }^{2}$ Emanuel Institute of Biochemical Physics, Russian Academy of Sciences, Kosygina st, 4, 119334, Moscow, Russia \\ ${ }^{3}$ Lomonosov Moscow State University, Faculty of Physics, Biophysics Department, 1/2 Leninskie gori, 119991, Moscow, Russia
}

\begin{abstract}
Purpose of study: to assess clinical outcomes of IVF programs after transfer of low quality microsurgically defragmented blastocysts.

Materials and methods: The study included 20 embryos of the 5-6th day of development, reached the blastocyst stage, whose trophectoderm was assessed as "C", due to presence of 15 to $35 \%$ of cytoplasmic fragments. Blastocysts underwent assisted hatching, followed by micromanipulation defragmentation. After defragmentation, the quality of embryos was re-assessed and used for embryo transfer (ET) or cryopreservation, followed by ET in cryocycle. The results of 20 clinical cases were analyzed with a known clinical outcome after the transfer of one defragmented blastocyst.
\end{abstract}

Results: 2 hours after defragmentation, trophectoderm of all 20 blastocysts was rated not lower than category "B". After ET the following was diagnosed: no pregnancy - 12 cases; early pregnancy loss - 2 cases; ongoing pregnancy resolved by live birth - 6 cases.

Conclusion: Defragmentation of low-quality blastocysts makes it possible to improve their morphological assessment. Clinical outcomes of IVF programs using defragmented blastocysts are comparable to those of good quality blastocyst transfer, including live births rate.

\section{Introduction}

The main criterion for assessing effectiveness of embryological stage of IVF programs is the presence and quantity of good quality embryos suitable for transfer. The morphological assessment of blastocysts is the most important criterion when choosing an embryo for transfer, since it largely reflects its viability $[1,2]$.

The presence of cytoplasmic fragments in perivitelline space of an embryo is recognized as the most common cause of a decrease in its viability and low morphological assessment [3]. In some cases, when, according to the results of IVF program, a patient does not have good quality embryos suitable for transfer, attempts are made to increase the viability of embryos by defragmenting them [4-6].

Defragmentation is a procedure for micromanipulation removal of cytoplasmic fragments, blastomeres that had stopped in development and cellular debris from the perivitelline space of an embryo. The aim of this work was to assess the clinical outcomes of IVF programs after transfer of low quality blastocysts via microsurgical defragmentation procedure.

\section{Materials and methods}

\section{Study design}

In the period from April to December 2019, the microsurgical defragmentation procedure was performed on 36 blastocysts of low quality obtained from 32 couples undergoing infertility treatment using ART methods at Altravita IVF Clinic, Moscow. In this and subsequent periods, embryo transfer (ET) or cryopreservation and storage of embryos were carried out with subsequent transfer in cryocycle, as well as assessment of the onset of pregnancy and analysis of the clinical outcomes of IVF programs. In January 2021, it became possible to obtain and analyze data on the clinical outcomes for 20 cases (after transfer of 20 blastocysts). The average age of women from whom embryos were obtained was 35.8 years (range 25 to 46 years). When selecting embryos for defragmentation, cause of infertility, woman's age and method of fertilization were not taken into account. Embryos obtained using donor oocytes, as well as in programs with PGD, were not included in the study. Criteria for inclusion of embryos in the experimental group were the following:

- no development of good quality blastocysts on the 5-6th suitable for ET or cryopreservation;

- development of low quality blastocyst on the 5-6th day, whose trophectoderm was assessed as "C" (according to Gardner) [1].

- volume of nuclear-free cytoplasmic fragments or blastomeres not included in the trophectoderm or ICM of the formed blastocyst was more than $15 \%$, but did not exceed $35 \%$ of embryo volume.

After ET, the following criteria were assessed: implantation rate, clinical and ongoing pregnancy rate, and live birth rate.

\section{Ovarian stimulation and oocyte retrieval}

Superovulation was induced by a "short" protocol using antGnRH from days $2-3$ of menstrual cycle using recombinant and / or urinary gonadotropins at daily dose of 150-300 IU. Ovulation was initiated

${ }^{\star}$ Correspondence to: Konstantin V Kirienko, Altravita IVF Clinic, 4A Nagornaya, 117186, Moscow, Russia, E-mail: kkiriyenko@rambler.ru

Key words: fragmentation of embryos, in vitro fertilization, embryo viability

Received: April 16, 2021; Accepted: August 03, 2021; Published: August 09, 2021 
by administration of hCG at a dose of 10,000 IU 34-36 hours before follicular puncture. Oocytes surrounded by layers of cumulus cells were obtained as a result of transvaginal puncture of follicles under ultrasound control 34-36 hours after the administration of an ovulatory dose of hCG.

\section{Fertilization}

For fertilization of the obtained oocytes, ICSI or IMSI procedure was used, depending on sperm parameters.

\section{Cultivation and assessment of embryo development}

Cultivation was carried out in a $\mathrm{CO}_{2}$-incubator at $36.7^{\circ} \mathrm{C}$ in humidified atmosphere with $6.0 \% \mathrm{CO}_{2}$. Embryos were individually cultured in microdroplets of Onestep medium (Vitromed) supplemented with $5 \mathrm{mg} /$ $\mathrm{ml}$ protein (SPS, Sage) under the layer of mineral oil. Zygotes and embryos were individually assessed on days 1, 3, 5, and 6 of development. Blastocyst quality was assessed according to Gardner [1].

\section{Defragmentation of embryos}

Blastocysts with trophectoderm assessed as " $\mathrm{C}$ " and fragmentation volume more than $15 \%$, but not more than $35 \%$ of embryo volume, underwent the microsurgical defragmentation procedure.

All microsurgical manipulations were performed on an inverted Leitz Fluovert FU microscope (Wild Leitz GmbH, Germany) using a Hoffman modulation contrast system and equipped with a set of Narishige micromanipulators (Narishige, Tokyo, Japan). At the first stage, assisted hatching was performed using a holding micropipette (Holding Pipette, Cook Medical) and the zona pellucida dissection micropipette (ZPD, Origio). From the side of maximum accumulation of cytoplasmic fragments, the zona pellucida was cut at $1 / 4$-circumference. After incision, cytoplasmic fragments, blastomeres that had stopped in development and cellular debris were removed from perivitelline space of an embryo using microinstruments for assisted hatching. In rare cases, when defragmentation was performed, the zona pellucida dissection micropipette may have been replaced with a biopsy micropipette (Biopsy Pipette, TPC) for greater operator convenience. For each embryo, the entire defragmentation procedure took 3-5 min. After defragmentation, blastocysts were cultured for 2 hours, then morphologically re-assessed and used for ET (5th day) or cryopreservation (5-6 days) followed by cryo-ET.

\section{ET, assessment of the onset and outcome of pregnancy}

ET was performed on the 5th day after follicular puncture (in ISO cycles) or after the start of progesterone therapy (in cryo-ET). One defragmented blastocyst was transferred into a patient's uterine cavity. The average number of embryos per transfer was 1.0 (one). Clinical pregnancy was diagnosed by the presence of gestational sac in the uterine cavity and registration of fetal heartbeat by ultrasound examination at $4-5$ weeks after ET.

Ongoing pregnancy rate was determined by ratio of the total number of clinical pregnancies (12 weeks or more) to the total number of transfers. At the end of gestational period, the number of live births was assessed.

\section{Results}

The results of 20 clinical cases with a known clinical outcome after transfer of one defragmented blastocyst in stimulation cycles (14 ETs) or cryo-cycles (6 cryo-ETs) were analyzed. Two hours after
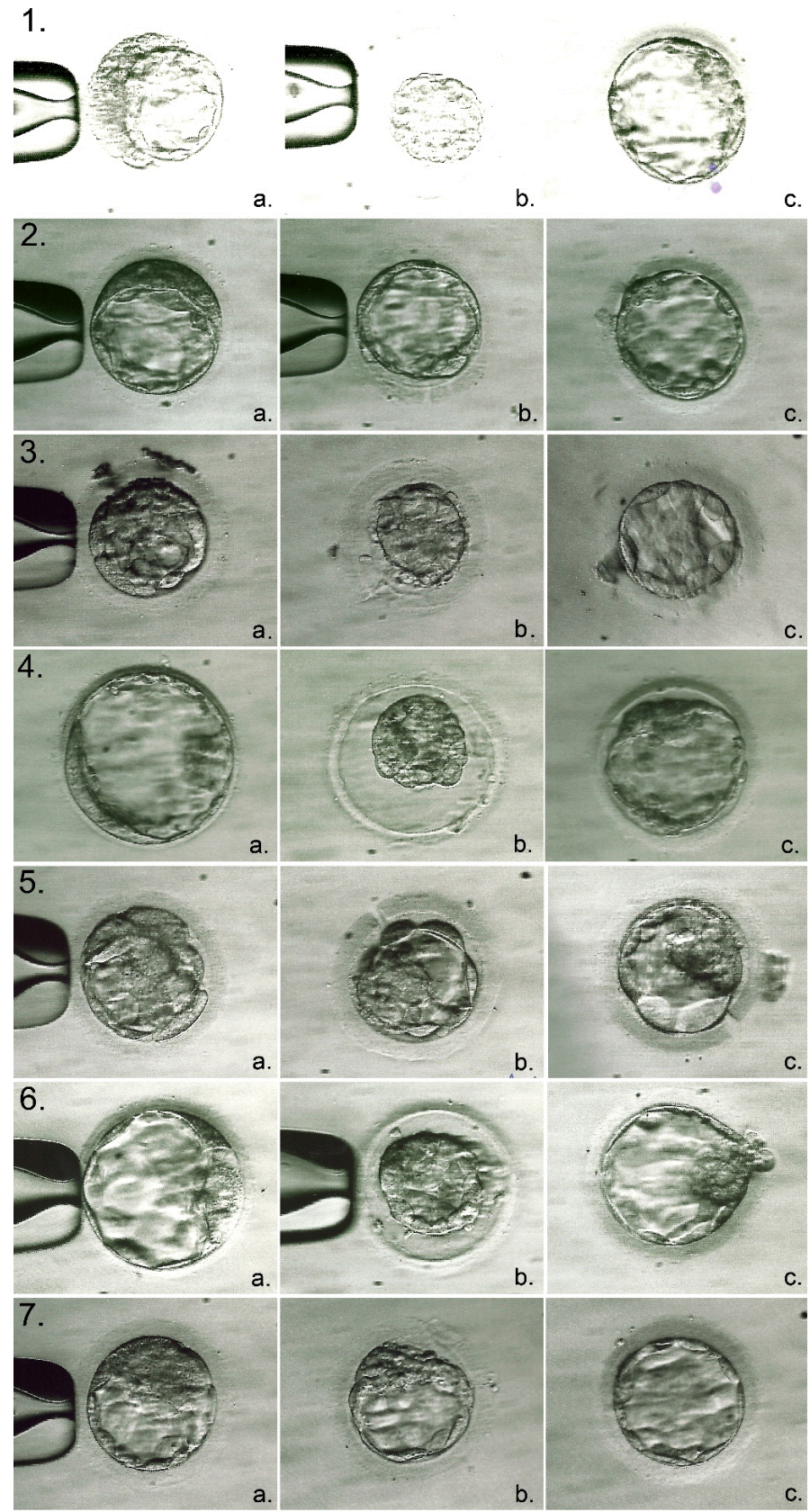

Figure 1. Blastocysts undergoing microsurgical defragmentation procedure (a. - before manipulations; b. - immediately after defragmentation; c. -2 hours after manipulations). As a result of blastocysts transfer No. 1,2, and 3, we could observe ongoingclinical pregnancy resolved by childbirth; No. 4-a pregnancy loss; No. 5, 6 and 7-no pregnancy.

microsurgical defragmentation, the trophectoderm of all 20 blastocysts was morphologically assessed not lower than category "B" (Figure 1).

After ET, the following was diagnosed:

- no pregnancy in 12 cases, including 8 with ET in ISO-cycles and 4 with cryo-ETs;

- clinical pregnancy in 8 cases,

- pregnancy loss in 2 cases, including 1 with ET in ISO-cycles and 1 with cryo-ET;

- ongoing pregnancy resolved by childbirth in 6 cases, including 5 with ETs in ISO-cycles and 1 with cryo-ET. 
Thus, clinical pregnancy rate was $40 \%(8 / 20)$ and ongoing pregnancy rate resolved by live birth was $75 \%(6 / 8)$. It should be noted that cumulatively for 2019 in Altravita IVF Clinic, these indicators were $34.2 \%$ and $65.1 \%$, respectively, when transferring good quality blastocysts rated not lower than "BB" (when transferring 1.1 embryos/ ET). In February 2020, a female healthy child was delivered at 37 weeks of gestation by cesarean section -the first child born as a result of defragmented low quality blastocysts transfer.

\section{Discussion}

In IVF practice, embryo transfer at the blastocyst stage is generally accepted standard [7]. The advantages of embryo transfer at the blastocyst stage include better synchronization between endometrium and embryo and ability to select an embryo with a higher implantation potential [8]. Gardner and Schoolcraft developed a blastocyst scoring system based on three parameters: blastocyst size, inner cell mass quality and trophectoderm quality [9].

When two good quality blastocysts $(\geq 3 \mathrm{AA})$ were transferred in ISO cycle, the implantation and clinical pregnancy rate were $69.9 \%$ and $86.8 \%$, respectively, while when using two blastocysts with quality lower than $3 \mathrm{AA}$, these indicators were only $28.1 \%$ and $43.8 \%$, respectively. In other words, the implantation of poorer quality blastocysts was more than 2 times lower than blastocysts with quality $\geq 3 \mathrm{AA}$ [1]. The same group of researchers obtained similar data in patients with a favorable prognosis after one blastocyst transfer: the proportion of embryo implantation and ongoing pregnancy rate was $60.9 \%$ [2]. In a retrospective study of 350 IVF cycles, Balaban and colleagues also found significant differences in the outcomes of IVF programs after transfer of blastocysts of varying quality [10].

Gray and colleagues modified the Gardner and Schoolcraft alphanumeric assessment scale to develop a blastocysts scoring system. According to their data, during embryo transfer in ISO cycles, quality of ICM and trophectoderm was a significant prognostic factor in assessing the onset of pregnancy. With a decrease of ICM in quality from "A" to "C", average blastocyst implantation rate decreased from $57 \%$ to $21 \%$, and in the case of a decrease in quality of trophectoderm, from $58 \%$ to $39 \%$, respectively [11].

A number of studies have shown that morphological assessment of blastocysts' trophectoderm makes it more likely to predict the pregnancy in IVF cycles [12,13]. In a retrospective study, Honnma and colleagues analyzed the contribution of each of the three components of blastocyst morphology assessment to clinical outcomes of IVF programs [14]. It was found that the quality of trophectoderm was statistically significantly associated with ongoing pregnancy and miscarriages rate. When transferring vitrified / thawed blastocysts with trophectoderm of quality $\mathrm{A}, \mathrm{B}$, and $\mathrm{C}$, the proportion of progressive clinical pregnancy was $46.6 \%, 34.1 \%$ and $23.7 \%$, respectively. On the contrary, neither the degree of blastocyst expansion nor the quality of ICM had a statistically significant effect on the clinical outcomes of IVF programs [14].

The reason for statistically significant correlation between trophectoderm quality and ongoing pregnancy and miscarriages rate is unclear. It is known that trophectoderm cells eventually form placenta, and cells of ICM form fetus. For this reason, it is believed that when selecting embryos for transfer, ICM morphology is more significant criterion for predicting pregnancy outcome than trophectoderm morphology, due to opinion that fetal cells are more important than placental cells. However, a number of studies have shown that trophectoderm morphology has a greater influence on the outcome of pregnancy than quality of ICM and degree of blastocyst expansion [12-14].
Some studies have shown that increased embryo fragmentation is associated with a decrease of implantation $[1,5,15]$ and pregnancy rate [15-17]. The data indicate that removal of fragments improves development of an embryo [18], and also increases the implantation rate $[5,15]$. It was found that, as a result of defragmented embryos transfer, the clinical outcomes of ART programs were similar [5] or better than those of embryos transfer from control groups [16].

Analysis of scientific publications showed that studies devoted to defragmentation effect on the clinical outcomes were carried out mainly using embryos at cleavage stage [4], in particular, on embryos of the 2 nd $[18,16]$ and 3rd day of development $[5,15]$.

We managed to find the only study where the object for defragmentation was human embryos at the blastocyst stage [19]. In this work, an overview of three clinical cases was presented on the transfer of defragmented blastocysts to patients in IVF programs with three clinical pregnancies. In our study, we report the birth of six children, obtained as a result of transfer of 20 low quality blastocysts after microsurgical defragmentation. To the best of our knowledge, this is the first study to report a live birth using low quality defragmented blastocyst for transfer.

It is believed that cytoplasmic fragmentation is a result of programmed cell death, leading to release of toxic substances that damage the surrounding cells or stop the cleavage of nearby blastomeres $[15,18]$. In addition, fragments can interfere with cell-tocell communication, disrupting the spatial orientation of blastomeres or limiting the space required for ongoing cleavage or compaction of an embryo. Research has shown that fragments cause secondary degeneration of nearby cells. Using transmission electron microscopy, it was found that blastomeres adjacent to cytoplasmic fragments show signs of vacuolization $[20,21]$. When these fragments are removed, the corresponding spatial organization of blastomeres in the embryo is restored, which leads to a more adequate formation of intercellular connections $[15,22]$. At the same time, fragmentation aspiration can improve microenvironment of blastomeres in the embryo by eliminating toxic effect of apoptotic cell fragments [16].

The main disadvantage of this and the above studies is their non-randomized nature. It remains to be determined which of the components of defragmentation procedure is of a decisive importance for improving the clinical outcomes of IVF programs: directly defragmentation or previous manipulations with the zona pellucida, ie, assissted hatching. Despite the fact that assisted hatching or complete removal of the zona pellucida in good quality blastocysts does not lead to increase of effectiveness of IVF programs [23], it can have a certain positive effect in case of embryos with low morphological assessment. Thus, further comparative prospective studies are needed to assess the role of embryo defragmentation in order to increase the ongoing pregnancy rate among patients undergoing IVF infertility treatment.

\section{Conclusion}

Defragmentation of low quality blastocysts makes it possible to improve their morphological assessment. Clinical outcomes of IVF programs using defragmented blastocysts are comparable to those of good quality blastocyst transfer, including live births rate.

\section{Contribution of authors}

Concept and design-Kirienko K.V.

Collection and processing of material-Kirienko K.V., Apryshko V.P., Voloshanenko V.V., Mironova A.G.

Text writing-K.V. Kirienko

Editing-S.A. Yakovenko 


\section{Declaration}

The authors report no financial or commercial conflicts of interest.

\section{References}

1. Gardner DK, Lane M, Stevens J, Schlenker T, Schoolcraft WB (2000) Blastocyst score affects implantation and pregnancy outcome: towards a single blastocyst transfer. Fertil Steril 73: 1155-1158. [Crossref]

2. Gardner DK, Surrey E, Minjarez D, Leitz A, Stevens J, et al. (2004) Single blastocyst transfer: a prospective randomized trial. Fertil Steril 81: 551-555.

3. Kirienko KV, Apryshko VP, Yakovenko SA (2020) Cytoplasmic fragmentation of human preimplantation embryos. Akusherstvo i Ginekologiya 11: 1-70 (in Russian).

4. Halvaei I, Khalili MA, Safari S, Esfandiari N (2015) Ongoing pregnancies following cosmetic micromanipulation of preimplantation embryos in patients with implantation failure. Case Rep Med 2015: 734793.

5. Keltz MD, Skorupski JC, Bradley K, Stein D (2006) Predictors of embryo fragmentation and outcome after fragment removal in in vitro fertilization. Fertil Steril 86: 321-324. [Crossref]

6. Chae-Kim JJ, Waggener K, Gavrilova-Jordan L (2020) Defragmentation of in-vitro fertilization blastocyst stage embryos leading to rescued blastocyst expansion and clinical pregnancy. Clin Obstet Gynecol Reprod Med 6: 1-3.

7. Meldrum DR (1999) Blastocyst transfer-a natural evolution. Fertil Steril 72: 216-217. [Crossref]

8. Olivennes F, Hazout A, Lelaidier C, Freitas S, Franchin R, et al. (1994) Four indications for transfer at the blastocyst stage. Hum Reprod 9: 2367-2373.

9. Gardner DK, Schoolcraft WB (1999) In vitro culture of human blastocyst. in: Jansen R Mortimer D. Towards reproductive certainty infertility and genetics beyond 1999 . Parthenon Press, Carnforth 378-388.

10. Balaban B, Urman B, Sertac A, Alatas C, Aksoy S, et al. (2000) Blastocyst quality affects the success of blastocyst-stage embryo transfer. Fertil Steril 74: 282-287.

11. Gray JE, Fritz MA, Berger DS (2016) Individual components of a Gardner's blastocyst grade and a summarized score are predictive of implantation for multiple but not single embryo transfers. Fertil Steril Poster Session 106: E209.
12. Zaninovic N, Berrios R, Clarke RN, Bodine R, Ye Z, et al. (2001) Blastocyst expansion, inner cell mass (ICM) formation, and trophectoderm (TM) quality: is one more important for implantation? Fertil Steril 76: S8.

13. Ahlstrom A, Westin C, Reismer E, Wikland M, Hardarson T (2011) Trophectoderm morphology: an important parameter for predicting live birth after single blastocyst transfer. Hum Reprod 26: 3289-3296. [Crossref]

14. Honnma H, Baba T, Sasaki M, Hashiba Y, Ohno H, et al. (2012) Trophectoderm morphology significantly affects the rates of ongoing pregnancy and miscarriage in frozen-thawed single-blastocyst transfer cycle in vitro fertilization. Fertil Steril 98: 361-367.

15. Alikani M, Cohen J, Tomkin G, Garrisi GJ, Mack C, et al. (1999) Human embryo fragmentation in vitro and its implications for pregnancy and implantation. Fertil Steril 71: 836-842. [Crossref]

16. Kim SG, Kim YY, Park JY, Kwak SJ, Yoo CS, et al. (2018) Early fragment removal on in vitro fertilization day 2 significantly improves the subsequent development and clinical outcomes of fragmented human embryos. Clin Exp Reprod Med 45: 122-128.

17. Ebner T, Yaman C, Moser M, Sommergruber M, Polz W, et al. (2001) Embryo fragmentation in vitro and its impact on treatment and pregnancy outcome. Fertil Steril 76: 281-285. [Crossref]

18. Eftekhari-Yazdi P, Valojerdi MR, Ashtiani SK, Eslaminejad MB, Karimian L (2006) Effect of fragment removal on blastocyst formation and quality of human embryos. Reprod BioMed Online 13: 823-832. [Crossref]

19. Chae-Kim JJ, Waggener K, Gavrilova-Jordan L (2020) Defragmentation of in-vitro fertilization blastocyst stage embryos leading to rescued blastocyst expansion and clinical pregnancy. Clin Obstet Gynecol Reprod Med 6: 1-3.

20. Halvaei I, Khalili MA, Esfandiari N, Safari S, Talebi AR, et al. (2016) Ultrastructure of cytoplasmic fragments in human cleavage stage embryos. J Assist Reprod Genet 33: 1677-1684. [Crossref]

21. Chi HJ, Koo JJ, Choi SY, Jeong HJ, Roh SI (2011) Fragmentation of embryos is associated with both necrosis and apoptosis. Fertil Steril 96: 187-192.

22. Alikani M (2007) The origins and consequences of fragmentation in mammalian eggs and embryos. Human Preimplantat Embryo Selection 51-78.

23. Kirienko K, Apryshko V, Naumova A, Kharitonova M, Klepukov A, et al. (2019) Mechanical zona pellucida removal of vitrified-warmed human blastocysts does not affect the clinical outcome. Reprod BioMed Online 39: 745-749.

Copyright: $\odot 2021$ Kirienko KV.This is an open-access article distributed under the terms of the Creative Commons Attribution License, which permits unrestricted use, distribution, and reproduction in any medium, provided the original author and source are credited. 